\section{Dieta mediterránea y sus efectos benéficos en la prevención de la enfermedad de Alzheimer}

\author{
ARNOLDO MIRANDA ${ }^{1, a}$, CAROLINA GÓMEZ-GAETE ${ }^{2, b}$, \\ SIGRID MENNICKENT ${ }^{2, a}$
}

\section{Role of Mediterranean diet on the prevention of Alzheimer disease}

Type 2 diabetes and obesity are possible risk factors for Alzheimer's disease and these can be modified by physical activity and changes in dietary patterns, such as switching to a Mediterranean diet. This diet includes fruits, vegetables, olive oil, fish and moderate wine intake. These foods provide vitamins, polyphenols and unsaturated fatty acids. This diet should be able to reduce oxidative stress. The inflammatory response is also reduced by unsaturated fatty acids, resulting in a lower expression and a lower production of pro-inflammatory cytokines. The Cardiovascular protection is related to the actions of polyphenols and unsaturated fatty acids on the vascular endothelium. The Mediterranean diet also can improve cardiovascular risk factors such as dyslipidemia, hypertension and metabolic syndrome. These beneficial effects of the Mediterranean diet should have a role in Alzheimer's disease prevention.

(Rev Med Chile 2017; 145: 501-507)

Key words: Alzheimer Disease; Diet, Mediterranean; Diet Therapy; Food.

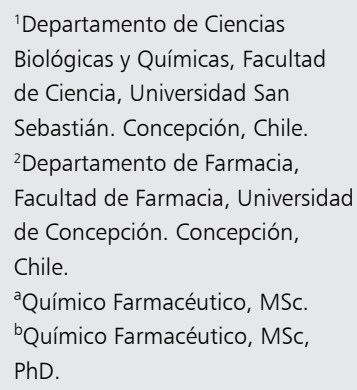

Fuente de apoyo financiero: Este artículo fue financiado por el Convenio de Desempeño UCO 1201, CONICYT y el programa de Magíster en Ciencias Farmacéuticas de la Universidad de Concepción.

Recibido el 9 de enero de 2016, aceptado el 1 de septiembre de 2016.

Correspondencia a: Arnoldo Miranda Tassara Departamento de Ciencias Biológicas y Químicas, Facultad de Ciencia, Universidad San Sebastián.

Lientur 1457, Concepción, Chile. arnoldo.miranda@uss.cl

\section{L}

a Enfermedad de Alzheimer (EA) es la causa más común de demencia en los adultos mayores afectando a $11 \%$ de ellos, la incidencia aumenta con la edad ${ }^{1}$ y en Chile la prevalencia de demencia en los mayores de 60 años es 10,4\% según cifras de la Encuesta Nacional de Salud 2009-2010, ubicándose dentro de las 10 primeras causas de muerte de este grupo etario ${ }^{2}$, siendo un tema importante en salud pública, ya que el envejecimiento se relaciona con el desarrollo de deterioro cognitivo y sumado al aumento en la esperanza de vida poblacional, gatilla que patologías como la EA vayan en aumento ${ }^{3}$.

En lo que respecta a su etiopatogenia, se ha establecido que la pérdida neuronal y sináptica se debe a la aparición de placas neuríticas y ovillos neurofibrilares a nivel cerebral, siendo estos elementos patológicos quienes repercuten en el desarrollo de la enfermedad y la diferencian de otros tipos de demencia, como la de origen vascular ${ }^{4}$.
Esta enfermedad cursa con deterioro cognitivo, sin embargo, este se caracteriza por comprometer solamente el estado cognitivo del paciente, sin ser un estado de gravedad tal que implique un diagnóstico de demencia o $\mathrm{EA}^{5}$.

El desarrollo de placas neuríticas está ligado al depósito extraneuronal de oligómeros de péptido beta-amiloide $(\mathrm{A} \beta)$, originado a partir de la proteína precursora de amiloide (amyloid precursor protein, APP) ubicada a nivel de la membrana citoplasmática, la cual normalmente durante su proteólisis por las secretasas $\alpha, \beta$ y $\gamma$, produce sustancias proteicas no tóxicas, pero en los pacientes con EA, debido a la acción de la secretasa $\beta$ y $\gamma$, se desencadena en mayor cuantía la producción del péptido $A \beta$ que posee efecto citotóxico (Figura 1). La placa neurítica es una estructura esférica que contiene $A \beta$ rodeada por neuritas distróficas, proteoglicanos, Apo $\varepsilon_{4}$, antiquimiotripsina $\alpha_{1}$ y otras proteínas ${ }^{4,6-11}$. 


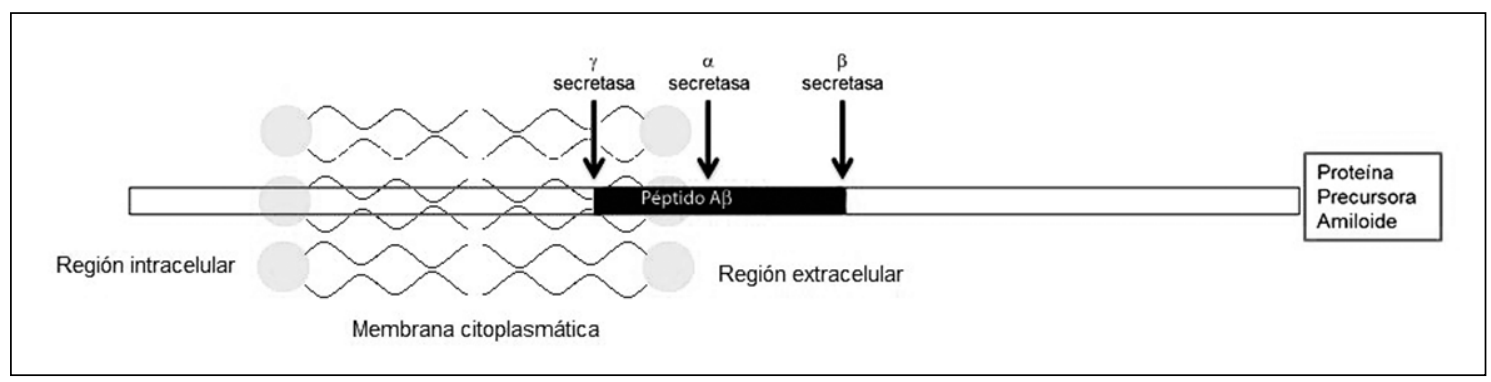

Figura 1. Representación de sitios de escisión de los diferentes tipos de secretasa en la Proteína Precursora Amiloide.

La aparición de los ovillos neurofibrilares es ocasionada por hiperfosforilación de la proteína tau, perteneciente a la familia de proteínas asociadas a microtúbulos (microtubule-associated proteins, MAPs), la cual participa del proceso de polimerización y estabilización de los microtúbulos neuronales y del proceso de transporte de ciertas sustancias a través del axón. Este proceso se encuentra relacionado con el péptido $A \beta$, ya que el depósito de éste induce la activación de vías de señalización neuronal implicadas en la fosforilación de tau, la cual cuando se hiperfosforila posee menor afinidad por los microtúbulos ocasionando disfunción neuronal y neurodegeneración $\mathrm{n}^{4,7-9,12,13}$.

La presencia de placas neuríticas y ovillos neurofibrilares activa procesos inflamatorios y apoptóticos, ocasionando muerte neuronal y, con ello, alteración de ciertos neurotransmisores claves en la progresión de la EA como acetilcolina y glutamato ${ }^{4}$.

\section{Factores de riesgo cardio-metabólicos de enfermedad de Alzheimer}

Los factores de riesgo asociados a la EA se clasifican en modificables y no modificables. Los factores modificables engloban a las enfermedades cardio-metabólicas como la diabetes mellitus tipo 2 (DM II), el síndrome metabólico (SM), dislipidemia, hipertensión arterial y la obesidad, ésta última relacionada con el sedentarismo, falta de actividad física y una dieta no equilibrada ${ }^{14-16}$.

Un meta-análisis realizado por Profenno et al. reafirmó que la DM II incrementa el riesgo de EA. Además, las complicaciones micro y macro vasculares asociadas a la DM II pueden aumentar el riesgo de padecer demencia vascular ${ }^{17}$. La hi- perglicemia e hiperinsulinemia se relacionan con una mayor acumulación de $\mathrm{A} \beta$ a nivel cerebral, lo cual ocurre debido a una disminución en la eliminación de $A \beta$ desde el cerebro. En este proceso participa la IDE (insulin degrading enzime), enzima que a su vez tiene como sustrato a la insulina, generándose una interferencia en la eliminación cerebral de $A \beta$, lo cual favorece su acumulación, incrementando el daño que éste ocasiona ${ }^{18}$.

En la DM II hay producción de compuestos de glicosilación avanzada, proceso que es perjudicial puesto que $\mathrm{A} \beta$ tiene mayor tendencia a agregarse cuando se encuentra glicosilado, potenciando así el daño cerebral. En estos pacientes se ha identificado un péptido denominado amilina, el cual tiende a depositarse y agregarse con $A \beta$ a nivel cerebral, formando placas y contribuyendo de esta manera a la progresión de la $\mathrm{EA}^{19}$.

Cifras extraídas del meta-análisis de Profenno et al., revelan que la obesidad aumenta en 59\% el riesgo de padecer $\mathrm{EA}^{20}$. Debido a la gran cantidad de tejido adiposo que presenta la mayoría de estos pacientes, ocurriría una mayor generación de citoquinas pro-inflamatorias, aumentando el estrés oxidativo.

Algunas adipoquinas como leptina, TNF- $\alpha$ e IL-6 son capaces de atravesar la barrera hematoencefálica afectando la función cerebral, predisponiendo al desarrollo de procesos patológicos $^{21,22}$. Se plantea que el tejido adiposo incide en la etiopatogenia del deterioro cognitivo ${ }^{23}$, lo cual se evidencia en un aumento en la fosforilación de tau en aquellos pacientes que presentan obesidad, DM II y disfunción de leptina, predisponiendo al desarrollo de $\mathrm{EA}^{24}$.

La dislipidemia aumenta el riesgo de padecer enfermedades cardiovasculares y cerebrovasculares, las cuales constituyen factores de riesgo 
de EA. En específico, se ha observado que en las personas adultas, entre 40 y 64 años, se relaciona la presión arterial elevada con un mayor riesgo de padecer deterioro cognitivo, demencia y $\mathrm{EA}^{25}$; esto se debería a que la hipertensión afectaría la integridad de la barrera hematoencefálica, provocando extravasación de proteínas hacia el tejido cerebral, conduciendo a daño celular, reducción de la función sináptica, apoptosis y mayor agregación de $\mathrm{A} \beta^{26}$.

Los factores de riesgo detallados anteriormente pueden ser manejados y prevenidos manteniendo un estilo de vida saludable, el cual se relaciona con una dieta adecuada y ejercicio. En base a lo observado a través de diversos estudios, se recomienda el consumo y una alta adherencia por una dieta mediterránea, tema al cual referencia este artículo de revisión.

\section{Dieta mediterránea y enfermedad de Alzheimer}

En la década de los 60, los resultados de un estudio epidemiológico desarrollado por Ancel Keys ${ }^{27}$ en donde se analizaron los aspectos dietéticos y el estilo de vida de las personas, demostraron que la población que habitaba en las cercanías del mar Mediterráneo presentaba menor incidencia de enfermedades cardiovasculares, lo cual se correlacionó con la alimentación, naciendo a partir de ese momento el concepto de dieta mediterránea ${ }^{28}$. Una dieta mediterránea se traduce en una alimentación rica en vegetales, frutas, legumbres, cereales y aceite de oliva, una baja ingesta de carnes rojas y grasas saturadas, moderado consumo de pescados y huevo, acompañando las comidas con especias e ingesta de vino en baja a moderada cantidad ${ }^{28}$.

Los hábitos alimenticios de las personas pueden repercutir de modo importante en la prevención o desarrollo de enfermedades como por ejemplo la $\mathrm{EA}^{29}$; es así como los resultados de una serie de estudios epidemiológicos realizados han relacionado una elevada adherencia a la dieta mediterránea con una mayor longevidad, menor prevalencia de enfermedades crónicas y una reducción en un 10\% del riesgo de eventos cardiovasculares $^{30}$, además de disminuir el riesgo de deterioro cognitivo y $\mathrm{EA}^{31}$ independiente de si la persona realiza o no actividad física con regularidad $^{32}$.

Por el lado contrario, una baja adherencia a la dieta mediterránea ha sido vinculada con una mayor predisposición a padecer deterioro cognitivo leve y $\mathrm{EA}^{33}$, razón por la cual se establece que esta dieta podría poseer un rol neuroprotector debido a su efecto antiinflamatorio, la diminución del estrés oxidativo y la protección cardiovascular que genera ${ }^{34}$.

Además de la dieta, se establece que la realización de actividad física en forma regular provoca un aumento del flujo sanguíneo, mejora la oxigenación y el aporte de glucosa a nivel cerebral, además de activar factores de crecimiento que promueven un aumento en la densidad capilar cerebral $^{3,35}$. La estimulación de la actividad cerebral e intelectual a través de juegos, puzzles, crucigramas y el fomento de la lectura tendría también un rol favorecedor en la protección contra el desarrollo de $\mathrm{EA}^{15}$.

En el año 2006, se realizó un estudio de tipo prospectivo buscando relacionar el consumo de dieta mediterránea con el riesgo de padecer EA, obteniéndose como relación una disminución entre 9 a 10\% del riesgo. Así como este estudio, existen otros que lograron relacionar de alguna manera la dieta y la EA, sin embargo, no todos los estudios han sido concluyentes en lograr correlaciones. Esto podría deberse a la dificultad de homologar los estudios, ya que las condiciones geográficas, culturales, sociodemográficas y ambientales no pueden ser controladas y podrían interferir con los resultados que se obtengan ${ }^{36,37}$.

\section{Efecto antiinflamatorio de la dieta mediterránea}

La inflamación juega un rol preponderante en la patogénesis tanto de la EA como de las enfermedades metabólicas. Diversos estudios han comprobado la presencia de niveles aumentados de marcadores inflamatorios en pacientes con EA, entre los cuales se encuentran el TNF- $\alpha$, IL-6 e IL- $1 \beta^{21,38}$.

El efecto antiinflamatorio observado en pacientes con alta adherencia a la dieta mediterránea se traduce en una disminución en marcadores inflamatorios a nivel plasmático como la proteína $\mathrm{C}$ reactiva ultrasensible $\left(\mathrm{PCR}_{\mathrm{us}}\right)$, la cual aumenta en presencia de placas seniles y fibrillas ${ }^{30,3)}$, y la IL- $6^{29}$.

El consumo de alimentos que aporten ácidos grasos omega 3 se asocia con la disminución del riesgo de demencia y deterioro cognitivo, debido 
a la incorporación de ácido eicosapentaenoico (EPA) y DHA a través de la dieta, los cuales presentan efectos antiinflamatorios y son fundamentales para un adecuado funcionamiento tanto neuronal y cerebral. El ácido docosahexaenoico (DHA) a través de la producción de neuroprotectina $\mathrm{D}_{1}$ es capaz de reprimir la expresión de genes implicados en el desarrollo de inflamación y de esta manera disminuye las citoquinas pro-inflamatorias a nivel plasmático ${ }^{14}$.Un estudio en el que se analizó una dieta rica en aceite de oliva, arrojó como resultado una disminución en la expresión de genes implicados en procesos inflamatorios y estrés oxidativo, de la misma manera los pacientes presentaron menores niveles plasmáticos de lípidos oxidados ${ }^{30}$, además presenta un efecto directo a nivel de tejido neural, siendo capaz de fomentar la plasticidad neuronal para procesos de aprendizaje, memoria y cognición. En pacientes con EA se ha observado un bajo nivel plasmático de DHA, y un estudio determinó que un aumento de éste se correlaciona de manera significativa con una disminución cercana al 39\% del riesgo de padecer EA ${ }^{14}$.

La ingesta de frutas y vegetales permite incorporar en el organismo compuestos con actividad antioxidante, los cuales se relacionan con una reducción de los niveles de PCR y homocisteí$\mathrm{na}^{39}$, siendo importante la presencia de menores concentraciones de este último compuesto, porque se ha verificado que niveles elevados de éste disminuyen la metilación del $\mathrm{ADN}$, favoreciendo la actividad de las secretasas $\beta$ y $\gamma$, incrementando de esta manera la producción del péptido $A \beta^{14}$.

\section{Dieta mediterránea y estrés oxidativo}

Los radicales libres y las especies reactivas del oxígeno que se generan en el organismo son neutralizados normalmente por moléculas endógenas $^{33}$, sin embargo, este balance antioxidante se altera en patologías relacionadas con la edad ${ }^{40}$, como la EA, favoreciendo una mayor producción de especies oxidativas las cuales se asocian con daño neuronal ${ }^{32}$, peroxidación lipídica y oxidación de enzimas relacionadas con la mantención de funciones de las neuronas y la glia ${ }^{41}$.

Los depósitos de $\mathrm{A} \beta$ se vinculan además con una disminución en las concentraciones de hierro y cobre a nivel cerebral, lo cual promueve estrés oxidativo y daño neuronal ${ }^{32}$. Junto con ello, este péptido es capaz de inhibir la cadena respiratoria, promoviendo disfunción mitocondrial y disminución de los niveles de ATP, desestabilizando los mecanismos de defensa antioxidante ${ }^{40}$.

La dieta mediterránea ayudaría a reducir el estrés oxidativo y la lipoperoxidación, por ejemplo, el aceite de oliva contiene micronutrientes los cuales presentan potenciales efectos antioxidantes, además de poseer ácidos grasos monoinsaturados los cuales tienen efectos benéficos sobre la presión arterial y el perfil lipídico, junto con ello son capaces de mejorar la DM y reducir el riesgo de trombosis ${ }^{39}$.

El alcohol podría acentuar el estrés oxidativo, por lo que es fundamental regular la cantidad en que es consumido; un meta-análisis de diversos estudios realizados logró comprobar que el alcohol, ingerido en forma moderada, se relaciona con un menor riesgo de desarrollar $\mathrm{EA}^{42}$. El efecto antioxidante del vino tinto se debería a la gran cantidad de polifenoles que contiene, entre ellos destaca el resveratrol que protegería a los vasos sanguíneos de los procesos inflamatorios, oxidativos, la agregación plaquetaria y formación de trombos, propias de la aterosclerosis ${ }^{39}$. Algunos estudios han logrado concluir que la vitamina $\mathrm{E}$, presente en semillas, nueces y vegetales de hojas verdes, podría reducir el riesgo de padecer demencia o $\mathrm{EA}^{43}$, además, esta vitamina combatiría el estrés oxidativo mediante la disminución de la lipoperoxidación y la apóptosis, en base a lo observado en estudios in vitro ${ }^{32}$, ya que los fosfolípidos son los principales afectados por la presencia de radicales libre y la vitamina $\mathrm{E}$, por su carácter liposoluble, es más afín con estos componentes celulares pudiendo prevenir su oxidación ${ }^{41,44}$. Mediante estudios in vitro e in vivo se confirmó que previene la hiperfosforilación de tau debido a que es capaz de interferir con la actividad de enzimas relacionadas con este proceso. Otros mecanismos que complementarían su acción son su rol como inhibidor de PKC, lo cual también previene la hiperfosforilación y por otro lado, cumple función reguladora de la expresión de genes relacionados con el estrés oxidativo ${ }^{45}$.

El consumo de alimentos ricos en carotenoides y vitamina $\mathrm{C}$ ayudaría en la protección contra la lipoperoxidación. Junto con ello, esta vitamina es capaz de disminuir la producción de nitrosaminas, contribuyendo a la disminución del estrés oxidativo ${ }^{32}$. En un análisis de 6 estudios realizados, se comprobó en al menos 4 de 
ellos que la ingesta de vitamina $\mathrm{C}$, betacaroteno, vitamina $\mathrm{E}$ y flavonoides disminuye la incidencia de demencia y $\mathrm{EA}^{14}$.

Junto a esto, diversos estudios avalan el rol protector de los ácidos grasos poliinsaturados dado que reducen el estrés oxidativo y favorecen la función neuronal y vascular, disminuyendo el riesgo de EA y demencia. Esto se debería a que son capaces de aumentar la actividad de la glutation reductasa y disminuir los niveles de especies reactivas del oxígeno, aumentando las defensas antioxidantes del organismo ${ }^{14,32}$.

\section{Dieta mediterránea y protección cardiovascular}

Los efectos cardioprotectores de la dieta mediterránea van de la mano con la disminución que ésta genera en el desarrollo de comorbilidades como hipertensión, dislipidemia o enfermedad coronaria $^{33}$.

Se ha visto que esta dieta es capaz de disminuir la glicemia, insulinemia y la resistencia a la insulina, además de mejorar la presión sistólica y disminuir los niveles de colesterol. El SM representa uno de los factores de riesgo para el desarrollo de EA, y se ha establecido que su prevalencia puede disminuirse a través de modificaciones de los hábitos nutricionales. La dieta mediterránea impacta de forma positiva al SM, ya que se ha demostrado que es capaz de disminuir la LDL oxidada y los lípidos plasmáticos, así como inhibir la activación de células inmunes y reducir la inflamación vascular, estos efectos sumados suponen una protección contra el desarrollo de aterosclerosis ${ }^{39}$.

El consumo de ácidos grasos poliinsaturados protege contra enfermedades vasculares, ya que mejoran algunos factores de riesgo asociados, son capaces de disminuir los triglicéridos, la presión arterial y mejorar el funcionamiento del miocardio $^{14}$, al contrario, una dieta que involucre el consumo de grasas saturadas y trans aumenta el riesgo de enfermedades cardiovasculares, DM II y con ello EA ${ }^{43,46}$.

\section{Conclusiones}

La EA es una patología cuya prevalencia se prevé vaya en aumento debido al envejecimiento poblacional a nivel mundial. Los tratamientos disponibles actualmente no son capaces de curar el cuadro, por lo que las estrategias no farmacológicas, relacionadas con los cambios en los estilos de vida juegan un rol fundamental a la hora de prevenir a largo plazo el desarrollo de esta enfermedad.

Es evidente que la disminución de los factores de riesgo cardiovasculares a los cuales se hace mención en esta revisión representaría un beneficio puesto que reduciría el riesgo de padecer EA, sin embargo, la dieta mediterránea ha demostrado ser una alimentación que posee efectos favorables que ayudan a disminuir las tasas de aparición de EA en la población debido a que, además de ayudar a mitigar los factores de riesgo modificables, se le atribuye una actividad neuroprotector a la cual va de la mano de sus efectos antioxidantes, antiinflamatorios y cardioprotectores.

El consumo de alimentos propios de esta dieta como frutas, vegetales, aceite de oliva, vino y pescados aportan a la dieta nutrientes fundamentales como vitaminas, carotenos, polifenoles y ácidos grasos insaturados los cuales contribuyen positivamente ejerciendo sus efectos biológicos en el organismo humano.

En base a los antecedentes disponibles en bibliografía, podría recomendarse el consumo y alta adherencia a una dieta mediterránea para disminuir complicaciones cognitivas en la población a largo plazo, pese a que no todos los estudios son concluyentes al respecto, sin embargo, deben ser considerados aquellos que demuestran evidencia sostenible en relación a los beneficios de esta dieta en la EA.

Si bien es difícil que los investigadores puedan controlar todas las variables de un estudio clínico sería importante, para poder lograr resultados certeros, contar con metodologías de trabajo claras en cuanto a las pautas de alimentación de los participantes, así como la realización de una selección adecuada de personas a incluir en los estudios, quienes debiesen ser uniformes en edad, raza y patologías de base, descartando a quienes posean factores de riesgo no modificables de EA.

Agradecimientos: Los autores agradecen al Convenio de Desempeño UCO 1201, al programa de Magíster en Ciencias Farmacéuticas de la Universidad de Concepción y a la beca CONICYT-PCHA/Magíster Nacional/2013-22131316. 


\section{Referencias}

1. Alzheimer's Association. 2014 Alzheimer's disease facts and figures. Alzheimers Dement 2014; 10 (2): e47-e92.

2. Gajardo J, Monsalves M. Demencia, un tema urgente para Chile. Rev Chil Salud Pública 2013; 17 (1): 22-5.

3. Bherer L, Erickson KI, Liu-Ambrose T. A Review of the Effects of Physical Activity and Exercise on Cognitive and Brain Functions in Older Adults. J Aging Res 2013; 2013: 657508 .

4. Maccioni CB, Arzola ME, Mujica L, Maccioni R. Nuevos paradigmas en el estudio de la patogénesis de la enfermedad de Alzheimer. Rev Chil Neuro-Psiquiat 2003; 41: 33-46.

5. Custodio N, Herrera E, Lira D, Montesinos R, Linares J, Bendezú L. Deterioro cognitivo leve: ¿dónde termina el envejecimiento normal y empieza la demencia? Anales de la Facultad de Medicina 2012; 73: 321-30.

6. Duyckaerts C, Delatour BT, Potier MC. Classification and basic pathology of Alzheimer disease. Acta Neuropathol 2009; 118 (1): 5-36.

7. García S, Coral VRM, Meza DE, Lucino CJ, Martínez SB, Villagómez OAJ. Enfermedad de Alzheimer: una panorámica desde su primera descripción hacia una perspectiva molecular. Med Int Mex 2009; 25 (4): 30012.

8. Jakob-Roetne R, Jacobsen H. Alzheimer's Disease: From Pathology to Therapeutic Approaches. Angew Chem Int Ed Engl 2009; 48 (17): 3030-59.

9. Leslie Crews EM. Molecular mechanisms of neurodegeneration in Alzheimer's disease. Hum Mol Genet 2010; 19 (1): R12-R20.

10. Palop JJ, Mucke L. Amyloid-[beta]-induced neuronal dysfunction in Alzheimer's disease: from synapses toward neural networks. Nat Neurosci 2010; 13 (7): 812-8.

11. Karran E, Mercken M, Strooper BD. The amyloid cascade hypothesis for Alzheimer's disease: an appraisal for the development of therapeutics. Nat Rev Drug Discov 2011; 10 (9): 698-712.

12. Ittner LM, Götz J. Amyloid-b and tau-a toxic pas de deux in Alzheimer's disease. Nat Rev Neurosci 2011; 12 (2): 67-72.

13. Barage $\mathrm{SH}$, Sonawane KD. Amyloid cascade hypothesis: Pathogenesis and therapeutic strategies in Alzheimer's disease. Neuropeptides 2015; 52: 1-18.

14. Otaegui-Arrazola A, Amiano P, Elbusto A, Urdaneta E, Martínez-Lage P. Diet, cognition, and Alzheimer's disease: food for thought. Eur J Nutr 2014; 53 (1): 1-23.

15. Reitz C, Brayne C, Mayeux R. Epidemiology of Alzheimer disease. Nat Rev Neurol 2011; 7 (3): 137-52.
16. Vagelatos NT, Eslick GD. Type 2 Diabetes as a Risk Factor for Alzheimer's Disease: The Confounders, Interactions, and Neuropathology Associated With This Relationship. Epidemiologic Reviews 2013.

17. Exalto LG, Whitmer RA, Kappele LJ, Biessels GJ. An update on type 2 diabetes, vascular dementia and Alzheimer's disease. Exp Gerontol 2012; 47 (11): 858-64.

18. Moreira PI. High-sugar diets, type 2 diabetes and Alzheimer's disease. Curr Opin Clin Nutr Metab Care 2013; 16 (4): 440-5.

19. Baram M, Atsmon-Raz Y, Ma B, Nussinov R, Miller $Y$. Amylin-A $\beta$ oligomers at atomic resolution using molecular dynamics simulations: a link between Type 2 diabetes and Alzheimer's disease. Phys Chem Chem Phys 2015; 18: 2330-8.

20. Profenno LA, Porsteinsson AP, Faraone SV. Meta-Analysis of Alzheimer's Disease Risk with Obesity, Diabetes, and Related Disorders. Biol Psychiatry 2010; 67 (6): 505-12.

21. Ferreira ST, Clarke JR, Bomfim TR, De Felice FG. Inflammation, defective insulin signaling, and neuronal dysfunction in Alzheimer's disease. Alzheimers Dement 2014; 10 (1): S76-S83.

22. Arnoldussen IAC, Kiliaan AJ, Gustafson DR. Obesity and dementia: Adipokines interact with the brain. Eur Neuropsychopharmacol 2014; 24 (12): 1982-99.

23. Misiak B, Leszek J, Kiejna A. Metabolic syndrome, mild cognitive impairment and Alzheimer's disease-The emerging role of systemic low-grade inflammation and adiposity. Brain Res Bull 2012; 89 (3-4): 144-9.

24. Platt TL, Beckett TL, Kohler K, Niedowicz DM, Murphy MP. Obesity, diabetes, and leptin resistance promote tau pathology in a mouse model of disease. Neuroscience 2016; 315: 162-74.

25. Campdelacreu J. Enfermedad de Parkinson y enfermedad de Alzheimer: factores de riesgo ambientales. Neurología 2014; 29 (9): 541-9.

26. Kalaria RN. Vascular basis for brain degeneration: faltering controls and risk factors for dementia. Nutr Rev 2010; 68 (suppl 2): S74-S87.

27. Keys A, Menotti A, Karvonem M, Aravanis C, Blackburn $\mathrm{H}$, Buzina R, et al. The diet and 15-year death rate in the seven countries study. Am J Epidemiol 1986; 124 (6): 903-15.

28. Feart C, Samieri C, Barberger-Gateau P. Mediterranean diet and cognitive health: an update of available knowledge. Curr Opin Clin Nutr Metab Care 2015; 18 (1): 51-62.

29. Scarmeas N, Stern Y, Tang M-X, Mayeux R, Luchsinger JA. Mediterranean diet and risk for Alzheimer's disease. Ann Neurol 2006; 59 (6): 912-21. 
30. Féart C, Samieri C, Allès B, Barberger-Gateau P. Potential benefits of adherence to the Mediterranean diet on cognitive health. Proc Nutr Soc 2012; 72 (01): 140-52.

31. Psaltopoulou T, Sergentanis TN, Panagiotakos DB, Sergentanis IN, Kosti R, Scarmeas N. Mediterranean diet, stroke, cognitive impairment, and depression: A meta-analysis. Ann Neurol 2013; 74 (4): 580-91.

32. Reitz C, Mayeux R. Alzheimer disease: Epidemiology, diagnostic criteria, risk factors and biomarkers. Biochem Pharmacol 2014; 88 (4): 640-51.

33. Gardener S, Gu Y, Rainey-Smith SR, Keogh JB, Clifton PM, Mathieson SL, et al. Adherence to a Mediterranean diet and Alzheimer/'s disease risk in an Australian population. Transl Psychiatry 2012; 2: e164.

34. Singh B, Parsaik AK, Mielke MM, Erwin PJ, Knopman DS, Petersen RC, et al. Association of Mediterranean diet with Mild Cognitive Impairment and Alzheimer's disease: A Systematic Review and Meta-Analysis. J Alzheimers Dis 2014; 39 (2): 271-82.

35. Yu F, Nelson NW, Savik K, Wyman JF, Dysken M, Bronas UG. Affecting Cognition and Quality of Life via Aerobic Exercise in Alzheimer's Disease. West J Nurs Res 2013; 35 (1): 24-38.

36. Yannakoulia M, Kontogianni M, Scarmeas N. Cognitive health and Mediterranean Diet: Just diet or lifestyle pattern? Ageing Res Rev 2015; 20: 74-8.

37. Nelson L, Tabet N. Slowing the progression of Alzheimer's disease; what works? Ageing Res Rev 2015; 23 (Pt B): 193-209.
38. Liu L, Chan C. The role of inflammasome in Alzheimer's disease. Ageing Res Rev 2014; 15: 6-15.

39. Pérez-López FR, Chedraui P, Haya J, Cuadros JL. Effects of the Mediterranean diet on longevity and age-related morbid conditions. Maturitas 2009; 64 (2): 67-79.

40. Silva T, Teixeira J, Remião F, Borges F. Alzheimer's Disease, Cholesterol, and Statins: The Junctions of Important Metabolic Pathways. Angew Chem Int Ed Engl 2013; 52 (4): 1110-21.

41. Huang W-J, Zhang XIA, Chen WW. Role of oxidative stress in Alzheimer's disease. Biomed Rep 2016; 4 (5): 519-22.

42. Titova OE, Ax E, Brooks SJ, Sjögren P, Cederholm T, Kilander L, et al. Mediterranean diet habits in older individuals: Associations with cognitive functioning and brain volumes. Exp Gerontol 2013; 48 (12): 1443-8.

43. Shah R. The Role of Nutrition and Diet in Alzheimer Disease: A Systematic Review. J Am Med Dir Assoc 2013; 14 (6): 398-402.

44. Schrag M, Mueller C, Zabel M, Crofton A, Kirsch WM, Ghribi O, et al. Oxidative stress in blood in Alzheimer's disease and mild cognitive impairment: A meta-analysis. Neurobiol Dis 2013; 59: 100-10.

45. La Fata G, Weber P, Mohajeri MH. Effects of Vitamin $\mathrm{E}$ on Cognitive Performance during Ageing and in $\mathrm{Al}-$ zheimer's Disease. Nutrients 2014; 6 (12): 5453-72.

46. Barnard ND, Bush AI, Ceccarelli A, Cooper J, de Jager CA, Erickson KI, et al. Dietary and lifestyle guidelines for the prevention of Alzheimer's disease. Neurobiol Aging 2014; 35 (Supplement 2): S74-8. 